\title{
Spectroscopic Study of the Planetary Nebula NGC 6210: Velocity Structure and Permitted Lines
}

\author{
Seong-Jae Lee, Jin-kyung Wi, and Siek Hyung* \\ Department of Earth Science, Chungbuk National University, Chungbuk 361-763, Korea
}

\begin{abstract}
Using the spectroscopic data secured with the Hamilton Echelle Spectrograph at Lick Observatory, we found the physical condition of the planetary Nebula NGC 6210. The spectral line profiles of the permitted and forbidden lines have been analyzed using IRAF and StarLink/Dipso. The hydrogen number densities $\left(\mathrm{N}_{\mathrm{H}}\right)$ are 2,000-20,000 $\mathrm{cm}^{-3}$, and the electron temperatures are 8,100-10,300 $\mathrm{K}$ based on the forbidden lines. The expansion velocities, derived from the Full Width at Half Maximum (FWHM) and the double peak of the line profiles, are in the range of 10 to $45 \mathrm{kms}^{-1}$. The expansion velocities imply a shell structure with an accelerated nebular gas. We also derived abundances from the permitted lines of CII, CIII, NII, NIII, OII, and OIII, which may have been formed through the fluorescence mechanism. NGC 6210 is likely to be evolved from a progenitor of more than $3 \mathrm{M}_{0}$, which had been born near the Galactic plane.

Keywords: Spectroscopic data, permitted line, line profile, planetary nebula, NGC 6210
\end{abstract}

\section{Introduction}

A low to intermediate star of $0.8-8$ Mo. spends most of its life span during the main sequence and the giant stages. When this star reaches the post-asymptotic giant branch (Post-AGB), it experiences mass-loss through thermal pulses and finally starts its life as a planetary nebula $(\mathrm{PN})$. Its life span at this final stage is very short, i.e., a couple of ten thousand years old. The central star of the PN (CSPN) becomes a hot white dwarf star and emits hot UV photons which ionize the surrounding gas. By analyzing the line profiles and intensities of $\mathrm{PN}$, one can obtain various hints on its formation or shaping history. The UV photons from the CSPN ionize atomic gases in the surrounding nebulous shell. As a result, the $\mathrm{H}$ and $\mathrm{He}$ gases produce the recombination lines, while the heavy elemental gases, such as $\mathrm{N}$ and $\mathrm{O}$, emit the forbidden lines. The line profiles are subject to broadening due to various factors. Once the subsidiary broadening factors are corrected, one can obtain the expansion velocities of the PN gas forming the shell, i.e., one of the most important clues for the shaping

*Corresponding author: hyung@chungbuk.ac.kr Tel: 82-43-261-2726

Fax: 82-43-271-0526 scenario of the PN. The line intensities themselves provide various important physical parameters and chemical abundances of the PN. Hence, one would also be able to acquire a hint for a chemical evolutionary history of intermediate stars.

The planetary nebula NGC 6210 displays an ellipsoidal appearance, while it has a skew-symmetric processing jet-like structure in a halo (Pottasch et al., 2009). Such an unusual point-symmetric morphology is often found in a Peimbert type I PN which evolved from a relatively massive progenitor (Peimbert, 1979). The CSPN is very bright and a hydrogen rich type $\mathrm{O}(\mathrm{H})$ star, i.e., $\mathrm{V}=12.66$ and $\mathrm{B}=12.44$ (Acker et al., 1992; Mendez, 1991). The hydrogen Zanstra temperature is relatively low, i.e., $50,000 \mathrm{~K}$, which is sufficient to ionize helium. Liu et al. (2004) and Kwitter and Henry (1998) derived abundances using the optical nebular spectra secured with the ground based telescope. The spectral wavelength dispersions of these data are $2 \AA /$ pixel or larger.

Most recently, Pottasch et al. (2009) analyzed the mid-infrared spectra secured with the Spitzer Space Telescope and the Infrared Space Observatory. They found the abundances for helium and oxygen which indicated that the CSPN was originally a rather low mass star $<1$ Mo. This result contradicts the expectation of the high massive progenitor origin found from an 
unusual appearance of the PN. Using the high dispersion spectral data $(0.28 \AA / \mathrm{pixel})$ observed with the Hamilton Echelle Spectrograph (HES) at Lick observatory, we investigated the expansion velocities of the shell gas to see whether there is any systematical change in the velocity structure of the expanding shell. We also investigated the permitted lines and derived their elemental abundances to compare with those of Kwitter and Henry (1998).

\section{HES Observations}

The spectroscopic observations had been carried out in 1995 July 4 (UT) and 2001 August 28 (UT) using the HES attached at the 3-m Shane telescope at Lick observatory. The whole nebular dimension is about $25 " \times 35$ ". We placed the slit entrance on the bright spot, 5" off-centered north-east and east of the CSPN, in 1995 and 2001: The nebular image rotates on the Coude focus of the 3-m Shane telescope, so we could not fix the slight direction on the image. The exposure times were 3 hours and 1 hour, respectively, in two separate observations. The employed CCD has 2048x 2048 pixels, which allowed us to secure various lines in the $3700-10,500 \AA$ wavelength range in one single exposure. The typical seeing was $1.5 "$. We chose a slit aperture area of $4 " \times 1.2^{\prime \prime}$ on the nebular image. The former 4" slit length corresponds to the spatial direction on the sky, while the latter 1.2" slit width corresponds to the wavelength dispersion. The wavelength dispersion is about $0.2 \AA$ pixel at $5000 \AA$.

As usual, we took exposures of a Th-Ar arc lamp to calibrate the wavelength, a dome-quartz lamp to fix a flat field, and standard stars of known energy distribution, i.e., HR7596 and HR9087 to correct the flux level. With the Image Reduction and Analysis Facilities (IRAF) echelle data reduction package provided by National Optical Astronomy Observatory, we extracted nebular spectral lines from raw images, employing Th-Ar maps to identify lines. The data reduction procedures are described in Hyung (1994). Fig. 1 shows the final scans of the spectra which show the lines of H 6563 and [NII] 6584. The H line
Table 1. Line intensities of NGC 6210 (I $(\mathrm{H})=100$.)

\begin{tabular}{|c|c|c|c|c|}
\hline Ion & $\lambda(\AA)$ & $\mathrm{I}_{\mathrm{obs}}$ (Ours) & $\mathrm{I}_{\mathrm{obs}}^{(1)}$ & $\mathrm{I}_{\mathrm{obs}}^{(2)}$ \\
\hline $\mathrm{HeI}$ & 4471 & 4.769 & 5.38 & 4.95 \\
\hline $\mathrm{HeI}$ & 5876 & 15.01 & 15.44 & $\ldots$ \\
\hline $\mathrm{HeI}$ & 6678 & 5.102 & 4.06 & 4.24 \\
\hline $\mathrm{HeI}$ & 7065 & 6.164 & 5.89 & $\ldots$ \\
\hline $\mathrm{HeI}+\mathrm{H} 8$ & 3889 & 20.07 & 19.75 & $\ldots$ \\
\hline HeII & 1640 & $\ldots$ & 7.92 & $\ldots$ \\
\hline HeII & 4686 & 2.533 & 1.21 & 2.71 \\
\hline $\mathrm{He}+[\mathrm{NeIII}]$ & 3968 & 15.86 & 45.60 & $\ldots$ \\
\hline CIII] & 1910 & $\ldots$ & 29.13 & $\ldots$ \\
\hline [NII] & 5755 & 0.274 & 0.60 & $\ldots$ \\
\hline [NII] & 6548 & 4.761 & 8.33 & 6.48 \\
\hline [NII] & 6584 & 15.35 & 28.39 & 22.70 \\
\hline [NIII] & 1750 & $\ldots$ & 2.07 & $\ldots$ \\
\hline [OIII] & 4363 & 5.567 & $\ldots$ & 1.42 \\
\hline$[\mathrm{OI}]$ & 6300 & 1.674 & 3.53 & 1.65 \\
\hline [OII] & 3726 & 18.04 & 44.93 & $\ldots$ \\
\hline [OII] & 7320,30 & 4.382 & 6.98 & $\ldots$ \\
\hline [OIII] & 4363 & 5.567 & 6.43 & 13.57 \\
\hline [OIII] & 4959 & 357.6 & 380.55 & 375.20 \\
\hline [OIII] & 5007 & 1062.3 & $\ldots$ & 847.39 \\
\hline [NeIII] & 3869 & 77.99 & 90.49 & $\ldots$ \\
\hline [SII] & 6716 & 0.862 & 2.64 & 1.74 \\
\hline [SII] & 6731 & 2.787 & 4.53 & 5.30 \\
\hline [SIII] & 6312 & 1.088 & 1.33 & 3.01 \\
\hline [SIII] & 9069 & 11.68 & 1.17 & $\ldots$ \\
\hline [SIII] & 9532 & 46.97 & 2.89 & $\ldots$ \\
\hline [CIIII] & 5538 & 0.428 & 0.55 & $\ldots$ \\
\hline [CIIII] & 5518 & 0.288 & 0.43 & $\ldots$ \\
\hline [ArIII] & 7135 & 11.89 & 13.08 & $\ldots$ \\
\hline [ArIII] & 7751 & 3.246 & 3.05 & $\ldots$ \\
\hline [ArIV] & 4740 & 1.889 & 1.51 & 2.11 \\
\hline [ArIV] & 4711 & 1.941 & 1.81 & 2.11 \\
\hline P8 & 9229 & 5.162 & 3.61 & $\ldots$ \\
\hline
\end{tabular}

(1) Kwitter and Henry (1998). (2) Phillips and Cuesta (1996)

is saturated in the year 1995's 3 hour exposure. We corrected the observed fluxes with an extinction coefficient $\mathrm{C}=0.36$ or $\mathrm{Av}=0.75 \quad[\mathrm{C}=\log \mathrm{I}(\mathrm{H}) / \mathrm{F}(\mathrm{H})]$. Here, $\mathrm{I}(\mathrm{H})$ is the intrinsic $\mathrm{H}$ line intensity and $\mathrm{F}(\mathrm{H})$ is the observed $\mathrm{H}$ flux. This extinction coefficient was derived from a comparison of the observed Balmer vs. Paschen line ratios. The wavelength dependent extinction parameter curve is adopted from Seaton (1979).

Table 1 lists the line intensities of NGC 6210 on the scale of $\mathrm{I}(\mathrm{H})=100.0$. The listed line intensities are combined from the 1995 and 2001 HES spectra. In this study, we will investigate the expansion velocities 

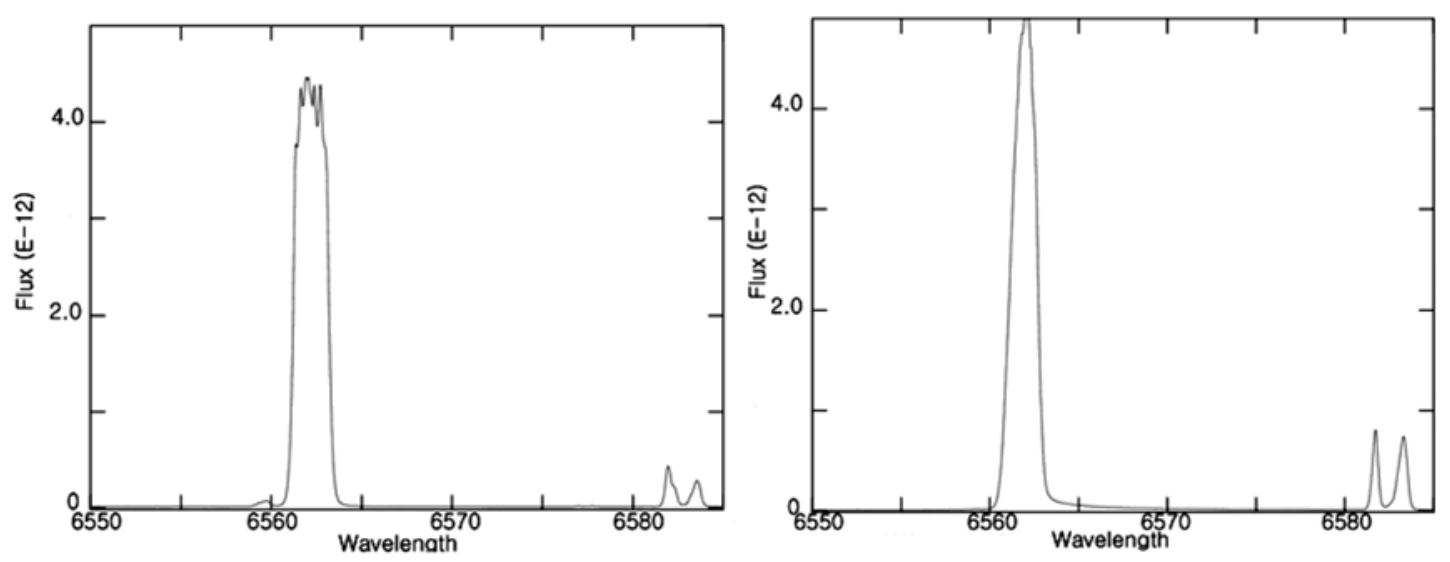

Fig. 1. HES spectra. (a) 1995 spectra (exposure $=3$ hrs, $\mathrm{H}$ saturated). (b) 2001 spectra (exposure $=1 \mathrm{hr}$ ). $\mathrm{H}$ shows a single gaussian profile, but [NII] 6584 shows a double peak.

obtained from the line profile analysis. Hence, we have only strong and strategically important diagnostic lines. The detailed analysis for the other weak lines will be given in another investigation. In general, our measurements agree with those by Kwitter and Henry (1998) and Phillips and Cuesta (1996). However, the spectra of our HES and Phillips and Cuesta (1996) seem to represent the central zone, while those of Kwitter and Henry (1998) appear to be from the whole nebular area: Our HES data indicate that high excitation lines, e.g., HeII, are stronger and the low excitation lines, e.g., [NII], are weaker than the Kwitter and Henry's measurement. We also quote the IUE spectra by Kwitter and Henry (1998) and Phillips and Cuesta (1996) in columns (4) and (5). Since our HES data have a high wavelength dispersion, most lines are well separated for the diagnostic studies. For example, [OII]7320, 7330 were not separable in Kwitter and Henry (1998) and Phillips and Cuesta (1996), but we were able to measure them separately with a high signal to noise ratio.

As discussed by Aller (1984), Pottasch (1984), Osterbrock (1989), and Sabbadin et al. (2004), one can determine the electron densities and temperatures, using the diagnostic lines. Table 2 lists the result, while Fig. 2 shows the diagnostic diagram. The electron densities are $N_{\varepsilon}=2,000-20,000 \mathrm{~cm}^{-3}$ and the electron temperatures are $T_{\varepsilon}=8,100-10,300 \mathrm{~K}$. We assumed that the $\left(N_{\varepsilon}=6,300 \mathrm{~cm}^{-3}, \quad T_{\varepsilon}=9,400 \mathrm{~K}\right)$ represents the physical condition of NGC 6210 .

\section{Line Profiles and Expansion Velocities}

Our HES data also allow us to study the kinematics of NGC 6210, which was not possible in the earlier investigations by Kwitter and Henry (1998) and Phillips and Cuesta (1996). Line profiles were analyzed using the IRAF and ESO StarLink/Dipso reduction package. Fig. 3 shows the decomposed line

Table 2. Electron temperatures and densities

\begin{tabular}{rccccccc}
\hline \hline Ion & lines used & $T_{\varepsilon}$ & $N_{\varepsilon}$ & Ion & lines used & $T_{\varepsilon}$ & $N_{\varepsilon}$ \\
\hline$[\mathrm{NII}]$ & $5755 / 6548+83$ & 9700 & & {$[\mathrm{SIII}]$} & $9069 / 6312+9531$ & 10300 & \\
{$[\mathrm{OII}]$} & $3726 / 3729$ & & 2070 & {$[\mathrm{ArIII}]$} & $5192 / 7736+51$ & 8100 & 43650 \\
& $3729+3726 / 7320+30$ & & 20400 & {$[\mathrm{ArIV}]$} & $4711 / 4740$ & 3160 \\
{$[\mathrm{OIII}]$} & $4363 / 4959+5007$ & 9360 & & & $4711+40 / 7171$ & 8900 \\
{$[\mathrm{SII}]$} & $6716 / 6731$ & & 2750 & {$[\mathrm{CIIII}]$} & $5518 / 5538$ & \\
{$[\mathrm{SIII}]$} & $9069 / 6312$ & 8660 & & & & \\
\hline
\end{tabular}




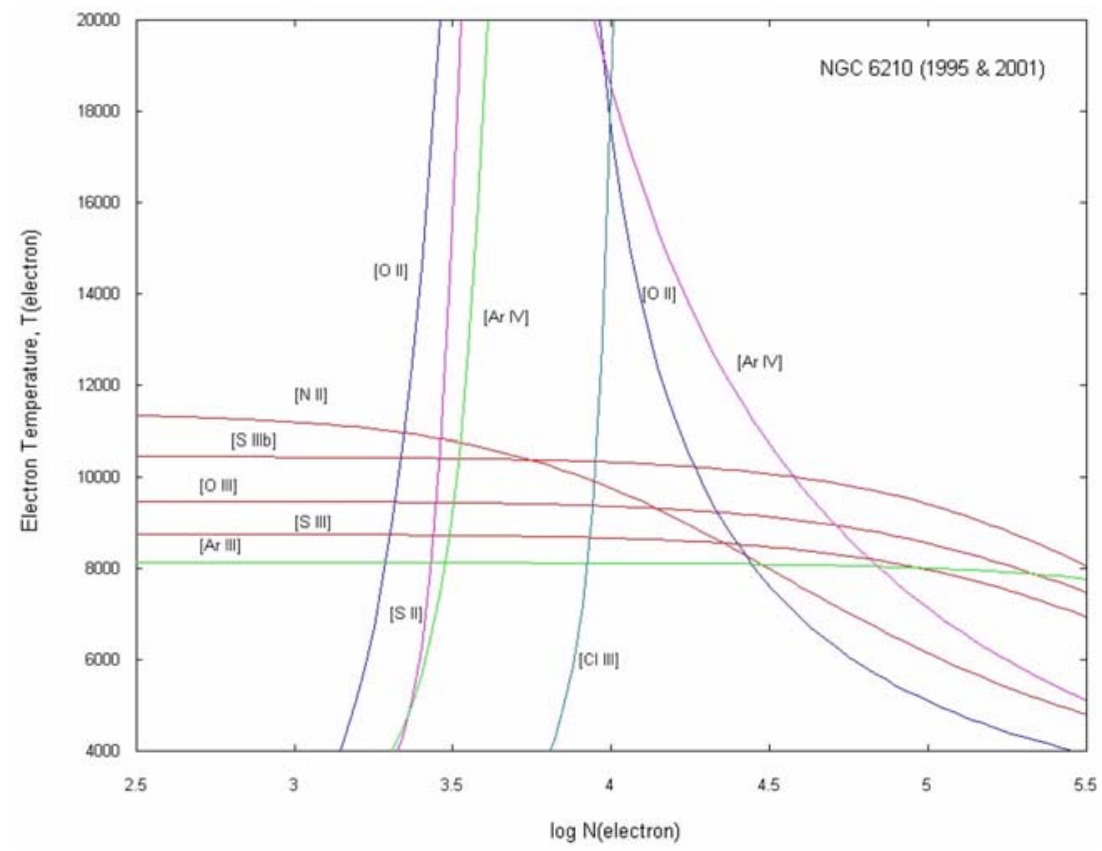

Fig. 2. Physical condition of NGC 6210 determined with diagnostic line ratios.

profiles of Paschen and Balmer lines. Although these two lines show a single peak, we were able to find two peaks. Both show the stronger line intensities in the receding red line profile. The stronger red line profile, appeared from chosen HES north-east (NE) or north $(\mathrm{N})$ position, represents the geometrical structure of the receding shell of the PN (i.e., rear part of the shell behind the CSPN).

Figure 4 shows two different types of line profiles. One would be able to measure expansion velocities in two different ways: 1) velocities based on the Full Width at Half Maximum (FWHM), $\Delta \lambda_{F W H M}$; 2) velocities based on two Gaussian peaks, $\Delta P \equiv \Delta \lambda=\lambda_{1}-$ $\lambda_{2}$, where $\lambda_{1}$ is the right peak wavelength and $\lambda_{2}$ is the other left peak wavelength. In the former single Gaussian profile case, one would be able to estimate the expansion velocities by measuring its FWHM.

The planetary nebula is known to have a stratification effect: The high excitation lines are mainly emitted from the inner zone of the PN shell, while the low excitation lines are radiated from the outer region. The low excitation lines mainly coming from the outer (hollow inside) shell would have a double Gaussian line profile, i.e., both blue and redshift components from the front and rear parts of the outer shell. The 1995 HES spectra show double Gaussian profiles mostly in low excitation lines. For example, the HI $\lambda 9545$ in Fig. 4 shows such a double peak Gaussian profile. Here, one would be able to measure two FWHMs, $\Delta \lambda_{\text {FWHM }}$, respectively, and the separation of two peaks, i.e., $\Delta P$. One would, however, be able to measure $\Delta P$ even in the case of one single Gaussian profile, whenever its signal to noise ratio is good enough, as in Fig. 3.

The Doppler shifted wavelength would allow us to derive the expansion velocity, i.e., $V_{\Delta P}=\frac{\Delta P}{\lambda} \times c$. Note that there are 4 other subsidiary broadening factors which must be eliminated in deriving the expansion velocities $\left.\left(V_{\text {exp }}\right): 1\right)$ thermal broadening, $V_{t h}$; 2) fine-structure, $\left.V_{f-s} ; 3\right)$ turbulence, $V_{t b}$; 4) instrumental broadening, $V_{\text {ins }}$. Details are given in Lee and Hyung (2007) and Sabbadin et al. (2006). Once These are known, the expansion velocity would be obtained as

$$
2 V_{\exp }=\left(V_{F W H M}^{2}-V_{t h}^{2}-V_{t b}^{2}-V_{f-s}^{2}-V_{i n s}^{2}\right)^{1 / 2} .
$$



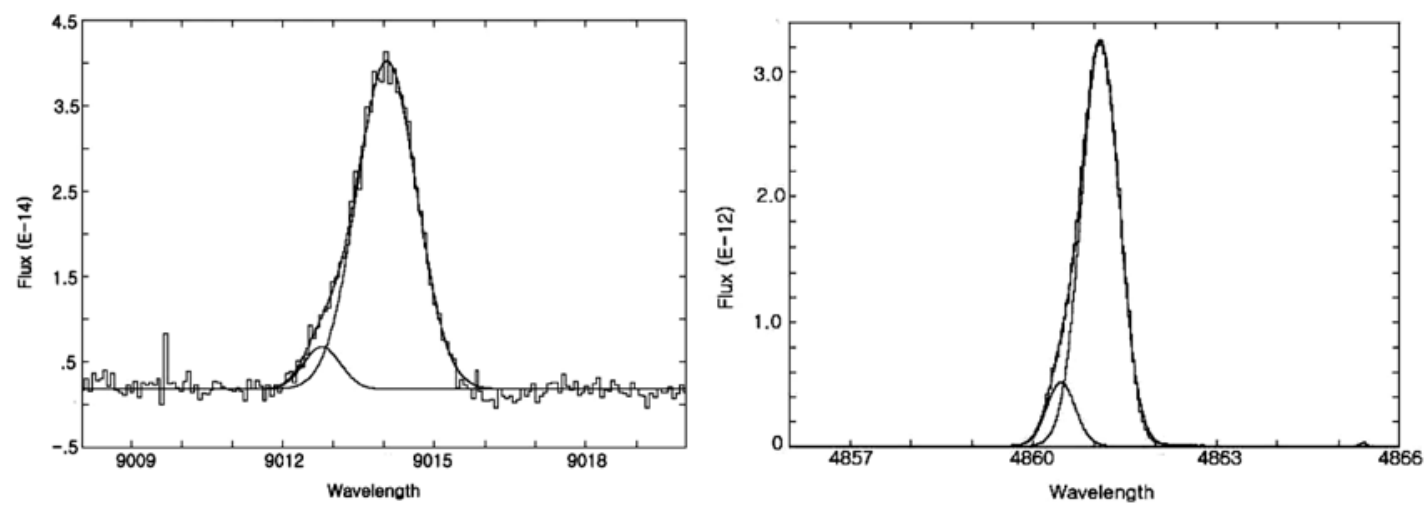

Fig. 3. Decomposed line profiles with Starlink/Dipso (2001). (a) HI 9014, (b) H 4861. Both spectra show stronger components in the red-shifted line profiles.
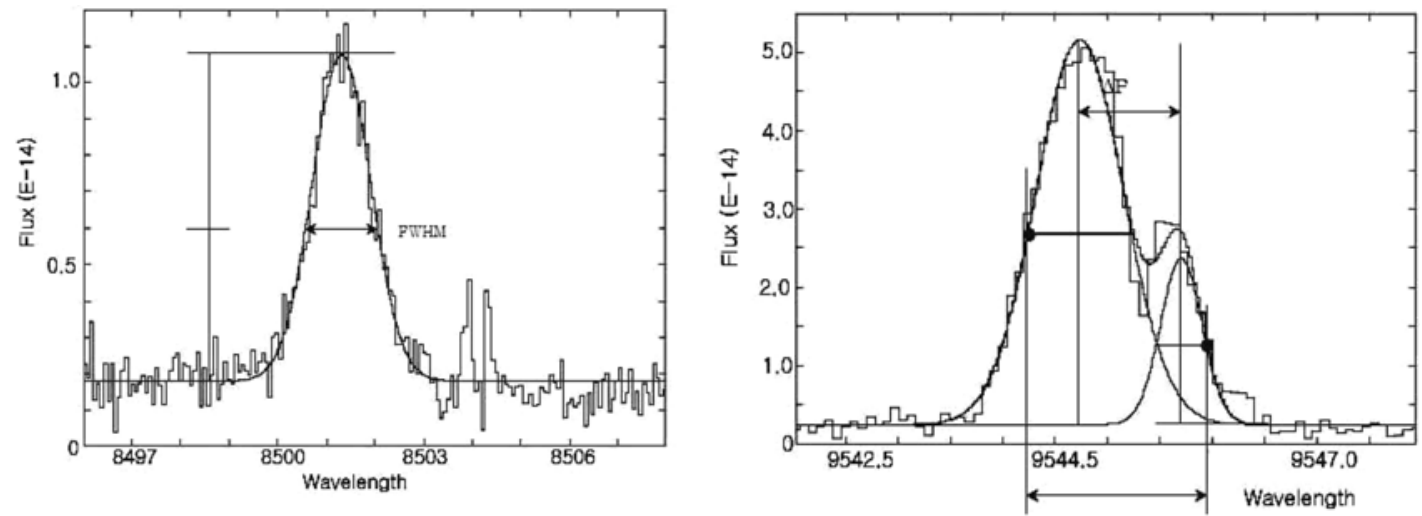

Fig. 4. FHWM of single and double gaussian and $\Delta P\left(=\lambda_{1}-\lambda_{2}\right)$. (a) 2001's Paschen line (P16 $\left.\lambda 8502\right)$. (b) 1995's Paschen line (P8 29545). The 2001's P16 shows a sing profile, while the 1995's P8 displays a double profile. The latter would gives more information on the nebular structure (see text).

Table 3 gives the derived expansion velocities. The expansion velocities, $V_{\text {exp }}$, derived from a single Gaussian FWHM, are about twice as large as those from a double Gaussian peak, $V_{\Delta R}$ As discussed in detail by Lee and Hyung (2007), this double gap occurs due to the difference of the shell expansion velocities at the inner and outer boundaries of the ionic shell. The expansion velocities in Table 3 are arranged relative to the decreasing order of the Ionization Potential (IP). Since the high excitation lines of a high IP emit close to the hot CSPN, such a decreasing order of expansion velocities may imply the radial variation of the shell.

Figure 5 shows the radial variation of the expansion velocities given in Table 3. The outflow velocities

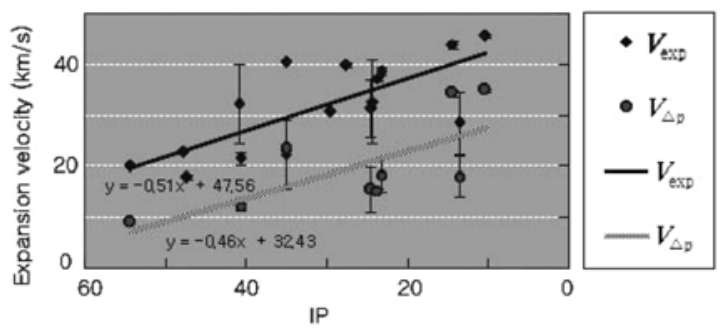

Fig. 5. Expansion velocities, $V_{\exp }$ and $V_{\Delta \beta}$ vs. IP. Since the higher IP corresponds to the inner zone, the plot implies that expansion velocities of the gas get accelerated.

appear to be accelerating from 10 to $45 \mathrm{kms}^{-1}$, as the gaseous material expands farther out. The slope of the accelerated outflow velocities is $0.5 \mathrm{kms}^{-1} / \mathrm{eV}$. Assuming that the nebtiar shell thickness is $0.05 \mathrm{pc}$ (for a 
616 Seong-Jae Lee, Jin-kyung Wi, and Siek Hyung

Table 3. Expansion velocities, $V_{\exp }$ and $V_{\Delta P}$

\begin{tabular}{|c|c|c|c|c|c|c|c|c|c|c|}
\hline$\lambda_{\text {obs }}(\AA)$ & Ion & FWHM $(\AA)$ & $\mathrm{V}_{\mathrm{FWHM}}$ & $\mathrm{V}_{\text {th }}$ & $V_{\text {f-s }}$ & $\mathrm{V}_{\text {turb }}$ & $2 V_{\exp }$ & $V_{\text {exp }}$ & $\Delta \mathrm{P}(\AA)$ & $\mathrm{V}_{\Delta \mathrm{P}}$ \\
\hline 9544.84 & $\mathrm{HI}$ & 1.72 & 53.99 & 20.94 & 7.62 & & 48.53 & 24.27 & 0.97 & 15.23 \\
\hline 9227.73 & H I & 1.66 & 53.93 & 20.94 & 7.62 & & 48.46 & 24.23 & 0.75 & 12.17 \\
\hline 9014.02 & $\mathrm{HI}$ & 1.54 & 51.28 & 20.94 & 7.62 & & 45.50 & 22.75 & 1.29 & 21.38 \\
\hline 8862.25 & H I & 1.47 & 49.59 & 20.94 & 7.62 & & 43.58 & 21.79 & 0.79 & 13.35 \\
\hline 8749.39 & H I & 1.54 & 52.66 & 20.94 & 7.62 & & 47.05 & 23.52 & 1.01 & 17.28 \\
\hline 8663.79 & $\mathrm{HI}$ & 1.58 & 54.70 & 20.94 & 7.62 & & 49.32 & 24.66 & 1.41 & 24.34 \\
\hline 8544.37 & H I & 2.00 & 70.23 & 20.94 & 7.62 & & 66.13 & 33.06 & 0.88 & 15.45 \\
\hline 8466.83 & $\mathrm{HI}$ & 1.58 & 56.15 & 20.94 & 7.62 & & 50.92 & 25.46 & 1.39 & 24.67 \\
\hline 6561.94 & $\mathrm{H} \alpha$ & 1.42 & 64.91 & 20.94 & 7.62 & & 60.45 & 30.22 & & \\
\hline 4861.06 & $\mathrm{H} \beta$ & 1.29 & 79.36 & 20.94 & 7.62 & & 75.75 & 37.88 & 0.65 & 19.93 \\
\hline 4340.10 & H I & 1.16 & 79.90 & 20.94 & 7.62 & & 76.32 & 38.16 & 0.51 & 17.49 \\
\hline \multirow[t]{3}{*}{4101.33} & $\mathrm{HI}$ & 1.08 & 79.25 & 20.94 & 7.62 & & 75.63 & 37.82 & 0.52 & 19.02 \\
\hline & & & & & & & mean & 28.65 & mean & 18.21 \\
\hline & & & & & & & $\sigma$ & 6.15 & $\sigma$ & 3.94 \\
\hline 3749.74 & $\mathrm{He} \mathrm{I}$ & 0.73 & 58.40 & 10.47 & 5 & & 56.68 & 28.34 & & \\
\hline 4471.07 & $\mathrm{He} \mathrm{I}$ & 1.20 & 80.31 & 10.47 & 5 & & 79.07 & 39.53 & 0.65 & 21.74 \\
\hline 5875.04 & $\mathrm{He} \mathrm{I}$ & 0.97 & 49.46 & 10.47 & 5 & & 47.42 & 23.71 & 0.44 & 11.23 \\
\hline 6677.90 & $\mathrm{He} \mathrm{I}$ & 1.66 & 74.41 & 10.47 & 5 & & 73.07 & 36.54 & 0.90 & 20.26 \\
\hline 7064.47 & $\mathrm{He} \mathrm{I}$ & 1.29 & 54.82 & 10.47 & 5 & & 52.98 & 26.49 & 0.60 & 12.76 \\
\hline \multirow[t]{3}{*}{7280.92} & $\mathrm{He} \mathrm{I}$ & 1.68 & 69.22 & 10.47 & 5 & & 67.77 & 33.89 & 0.61 & 12.59 \\
\hline & & & & & & & mean & 31.42 & mean & 15.72 \\
\hline & & & & & & & $\sigma$ & 5.65 & $\sigma$ & 4.37 \\
\hline 4685.15 & $\mathrm{He} \Pi$ & 0.67 & 42.80 & 10.47 & 5 & & 40.42 & 20.21 & 0.29 & 9.19 \\
\hline 4266.67 & $\mathrm{C} \Pi$ & 0.71 & 49.96 & 6.05 & 5 & & 48.69 & 24.35 & \multirow{18}{*}{1.54} & \multirow{18}{*}{36.35} \\
\hline 6461.24 & $\mathrm{C \Pi}$ & 1.91 & 88.67 & 6.05 & 5 & & 87.97 & 43.98 & & \\
\hline \multirow[t]{3}{*}{6577.22} & $\mathrm{C} \Pi$ & 1.34 & 61.29 & 6.05 & 5 & & 60.27 & 30.13 & & \\
\hline & & & & & & & mean & 32.82 & & \\
\hline & & & & & & & $\sigma$ & 8.24 & & \\
\hline 4646.87 & $\mathrm{CIII}$ & 0.73 & 47.05 & 6.05 & 5 & & 45.71 & 22.85 & & \\
\hline 5679.35 & $\mathrm{N \Pi}$ & 1.19 & 62.75 & 5.60 & 5 & & 61.79 & 30.90 & & \\
\hline 4096.65 & NIII & 0.51 & 37.69 & 5.60 & 5 & & 36.07 & 18.04 & & \\
\hline 4102.93 & NIII & 0.50 & 36.75 & 5.60 & 5 & & 35.09 & 17.54 & & \\
\hline \multirow[t]{3}{*}{4640.09} & NIII & 0.58 & 37.76 & 5.60 & 5 & & 36.14 & 18.07 & & \\
\hline & & & & & & & mean & 18.06 & & \\
\hline & & & & & & & $\sigma$ & 0.43 & & \\
\hline 6363.61 & O I & 2.011 & 94.80 & 5.24 & 5 & & 94.19 & 47.10 & & \\
\hline 4071.77 & $\mathrm{O} \Pi$ & 0.62 & 45.76 & 10.47 & 5 & & 43.55 & 21.77 & & \\
\hline 4638.34 & $\mathrm{O \amalg}$ & 0.75 & 48.72 & 10.47 & 5 & & 46.65 & 23.32 & & \\
\hline \multirow[t]{3}{*}{4648.63} & $\mathrm{O} \Pi$ & 0.73 & 46.91 & 10.47 & 5 & & 44.75 & 22.37 & & \\
\hline & & & & & & & mean & 22.49 & & \\
\hline & & & & & & & $\sigma$ & 6.79 & & \\
\hline
\end{tabular}

distance of $1 \mathrm{kpc}$, see Acker et al., 1992), one can guess that it has been accelerated from $10 \mathrm{kms}^{-1}$ to $V_{\exp }$ for $45 \mathrm{kms}^{-1}$ erhile travelling the shell radius of $0.05 \mathrm{pc}\left(10^{\prime \prime}\right)$. Another alternative interpretation is that the gas ejected quickly in the beginning, e.g., a few thousand years ago, but recently it might have become slow. The detailed discussion is beyond the limit of the current study. The former interpretation of an accelerated outflow has been accepted more widely than the latter interpretation, though.

It has been a long standing problem that the abundance derived from the recombination lines is 
Table 3. Continued

\begin{tabular}{|c|c|c|c|c|c|c|c|c|c|c|}
\hline$\lambda_{\text {obs }}(\AA)$ & Ion & FWHM $(\AA)$ & $\mathrm{V}_{\mathrm{FWHM}}$ & $\mathrm{V}_{\text {th }}$ & $V_{\text {f-s }}$ & $\mathrm{V}_{\text {turb }}$ & $2 V_{\text {exp }}$ & $V_{\text {exp }}$ & $\Delta \mathrm{P}(\AA)$ & $\mathrm{V}_{\Delta \mathrm{P}}$ \\
\hline 4362.78 & [OIII] & 1.19 & 82.03 & 5.24 & & 5 & 81.32 & 40.66 & 0.70 & 24.00 \\
\hline 4958.43 & [OIII] & 1.34 & 81.28 & 5.24 & & 5 & 80.56 & 40.28 & 0.76 & 23.14 \\
\hline \multirow[t]{3}{*}{5006.25} & [OIII] & 1.38 & 82.81 & 5.24 & & 5 & 82.11 & 41.05 & 0.78 & 23.31 \\
\hline & & & & & & & mean & 40.66 & mean & 23.48 \\
\hline & & & & & & & $\sigma$ & 0.31 & $\sigma$ & 0.37 \\
\hline 5754.39 & [NII] & 1.68 & 87.58 & 5.60 & & 5 & 86.90 & 43.45 & 1.30 & 33.86 \\
\hline 6547.76 & [NII] & 1.92 & 88.06 & 5.60 & & 5 & 87.38 & 43.69 & 1.49 & 34.20 \\
\hline \multirow[t]{3}{*}{6583.26} & [NII] & 1.99 & 90.77 & 5.60 & & 5 & 90.11 & 45.06 & 1.55 & 35.32 \\
\hline & & & & & & & mean & 44.06 & mean & 34.46 \\
\hline & & & & & & & $\sigma$ & 0.71 & $\sigma$ & 0.62 \\
\hline 6716.07 & {$[\mathrm{SII}]$} & 2.08 & 92.73 & 3.70 & & 5 & 92.18 & 46.09 & 1.56 & 34.84 \\
\hline \multirow[t]{3}{*}{6728.95} & [SII] & 2.04 & 91.15 & 3.70 & & 5 & 90.59 & 45.29 & 1.59 & 35.37 \\
\hline & & & & & & & mean & 45.69 & mean & 35.10 \\
\hline & & & & & & & $\sigma$ & 0.40 & $\sigma$ & 0.26 \\
\hline 9067.57 & [SIII] & 2.31 & 76.55 & 3.70 & & 5 & 75.88 & 37.94 & 1.32 & 21.83 \\
\hline \multirow[t]{3}{*}{9529.81} & [SIII] & 2.53 & 79.70 & 3.70 & & 5 & 79.06 & 39.53 & 0.96 & 15.17 \\
\hline & & & & & & & mean & 38.73 & mean & 18.50 \\
\hline & & & & & & & $\sigma$ & 0.79 & $\sigma$ & 3.33 \\
\hline 8577.29 & [ClII] & 2.60 & 90.92 & 3.51 & & 5 & 90.37 & 45.18 & 2.12 & 37.12 \\
\hline 5517.52 & [ClIII] & 1.41 & 76.39 & 3.51 & & 5 & 75.73 & 37.86 & 0.56 & 15.14 \\
\hline \multirow[t]{3}{*}{5537.37} & [ClIII] & 1.37 & 74.35 & 3.51 & & 5 & 73.67 & 36.84 & 0.58 & 15.79 \\
\hline & & & & & & & mean & 37.35 & mean & 15.47 \\
\hline & & & & & & & $\sigma$ & 0.51 & $\sigma$ & 0.32 \\
\hline 5322.62 & [ClIV] & 0.91 & 51.46 & 3.51 & & 5 & 50.48 & 25.24 & & \\
\hline 7529.48 & [ClIV] & 1.39 & 55.47 & 3.51 & & 5 & 54.56 & 27.28 & 0.78 & 15.62 \\
\hline \multirow[t]{3}{*}{8044.46} & [ClIV] & 0.98 & 36.61 & 3.51 & & 5 & 35.21 & 17.61 & 0.69 & 12.81 \\
\hline & & & & & & & mean & 23.37 & mean & 14.21 \\
\hline & & & & & & & $\sigma$ & 4.16 & $\sigma$ & 1.40 \\
\hline 7134.93 & [ArIII] & 1.90 & 79.67 & 3.31 & & 5 & 79.04 & 39.52 & 1.90 & 39.83 \\
\hline \multirow[t]{3}{*}{7750.70} & [ArIII] & 2.12 & 81.89 & 3.31 & & 5 & 81.29 & 40.64 & 1.27 & 24.60 \\
\hline & & & & & & & mean & 40.08 & mean & 32.21 \\
\hline & & & & & & & $\sigma$ & 0.56 & $\sigma$ & 7.62 \\
\hline 4710.87 & [ArIV] & 0.66 & 42.06 & 3.31 & & 5 & 40.86 & 20.43 & 0.36 & 11.62 \\
\hline \multirow[t]{3}{*}{4739.93} & [ArIV] & 0.74 & 46.96 & 3.31 & & 5 & 45.89 & 22.95 & 0.42 & 13.23 \\
\hline & & & & & & & mean & 21.69 & mean & 12.42 \\
\hline & & & & & & & $\sigma$ & 1.26 & $\sigma$ & 0.80 \\
\hline 3868.37 & [NeIII] & 0.65 & 50.20 & 4.68 & & 5 & 49.09 & 24.54 & & \\
\hline \multirow[t]{3}{*}{3967.03} & [NeIII] & 1.07 & 81.21 & 4.68 & & 5 & 80.53 & 40.27 & 0.62 & 23.48 \\
\hline & & & & & & & mean & 32.40 & & \\
\hline & & & & & & & $\sigma$ & 7.86 & & \\
\hline 5345.40 & [KrIV] & 1.62 & 90.91 & 2.90 & & 5 & 90.38 & 45.19 & & \\
\hline 6101.56 & [KIV] & 0.63 & 31.05 & 3.35 & & 5 & 29.40 & 14.70 & & \\
\hline
\end{tabular}

$\mathrm{V}_{\text {inst }}=7.95 \mathrm{kms}^{-1}$ is considered for all lines.

different from that from the forbidden lines. Hence, the comparison of the expansion velocities measured from the recombination lines with those from the same ionization stage of forbidden lines is important, since it would give a clue as to whether these two are originated from the same ion. As in Table 3, the derived expansion velocities are not the same in two cases, i.e., $22.49 \pm 6.79$ (OII) vs. $40.6 \pm 60.31 \mathrm{kms}^{-1}$ 

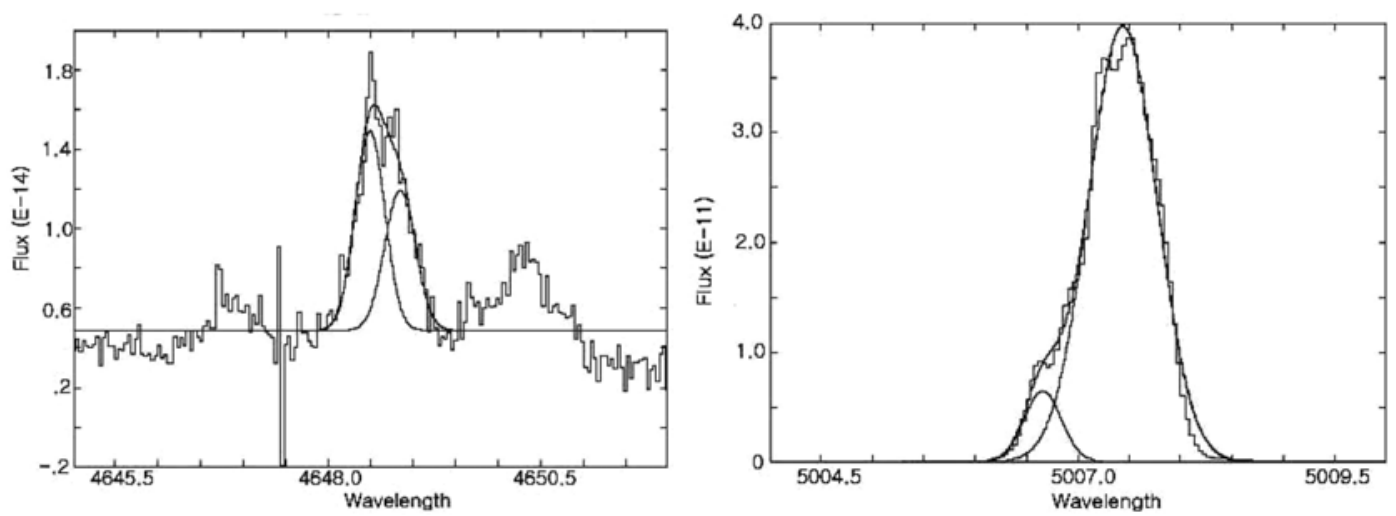

Fig. 6. OII $\lambda 4648$ (left) and [OIII] $\lambda 5007$ (right) line profile. Disagreement between the two line profiles indicates that they are from different kinematical structure.

([OIII]). Figure 6 shows the line profile of these two lines. In spite of a large difference in the quality of these two lines, one can clearly see they are formed in a different mechanism or different zone. If the OII lines are formed through the recombination mechanism like HI, HeI, HeII lines, both OII and [OIII] lines are from the same $\mathrm{O}^{2+}$ ion in the same zone. The slow expansion indicated by the former OII line implies that this has formed through another radiation source close to the central region where the expansion velocity is slow. The OII lines may not actually be from the $\mathrm{O}^{2+}$ ion.

The similarity of the expansion velocity of OII lines to the HeII implies that the OII lines might have been emitted in the central zone where the HeII lines are formed. The OII lines and other permitted CII, NII, and NIII lines might have been from other ions, e.g., HeII or stellar continuum, through a fluorescence or a resonance mechanism. In Sec. 4, we will derive abundances based on permitted lines and compare them with those given in the literature.

\section{Abundances}

Contrary to the Sun, PNs do not show many permitted lines except for OII lines. However, we are able to identify many permitted lines in NGC 6210 . These are CII, CII, NII, NIII, and OIII in addition to HI, HeI, and HeII, and OII lines. The other PNs which have permitted lines are NGC 7009, NGC 7662, NGC 6803, etc. (Choi et al., 2008). Using the recombination coefficients (Liu et al., 1995; Péquignot et al., 1991), the chemical abundances based on the permitted lines can be derived as

$$
\frac{\mathrm{O}^{2+}}{\mathrm{H}^{2+}}=\frac{\lambda(\AA)}{4861} \frac{\alpha_{e f f}\left(H_{\beta}\right)}{\alpha_{e f f}(\lambda)} \frac{I(\lambda)}{I\left(H_{\beta}\right)}
$$

where $\alpha_{e f f}$ is

$$
\alpha=10^{-13} z \frac{a t^{b}}{1+c t^{d}}
$$

Here, $z$ is a charge of element; $t=10^{-4} T_{\varepsilon} / z^{2} ; a, b$ and $c$ are from Péquignot et al. (1991). For example, $\alpha_{\text {eff }}\left(H_{\beta}\right)$ will be given as

$$
\alpha_{e f f}\left(H_{\beta}\right)=10^{-13} \times 1 \times \frac{0.668 \times 0.94^{-0.507}}{1+1.221 \times 0.94^{0.653}}=0.317 \times 10^{-13}
$$

assuming $T_{\varepsilon}=9,400 \mathrm{~K}$. The effective recombination coefficients for $\lambda 4072 \AA$ and $\lambda 4075 \AA$ (transitions, $J \rightarrow J^{\prime}=4 \mathrm{f}-3 \mathrm{~d}$ and $3 \mathrm{~d}-3 \mathrm{p}$ ) are from

$$
\alpha\left(J, J^{\prime}\right)=(2 J+1) B\left(J, J^{\prime}\right) \alpha
$$

where $B\left(J, J^{\prime}\right)=0.865(\lambda 4072 \AA)$ and $1.00(\lambda 4075 \AA)$; $\alpha=10^{-14} a t^{b}\left[1+c(1-t)+d(1-t)^{2}\right] . a, b, c$, and $d$ are given in Liu et al. (1995), that is, $a=0.876, b=-0.74621$, $\mathrm{c}=0.15710$, and $\mathrm{d}=0.07059$ for our case. Hence, we are able to get $\alpha(4072)=0.924 \times 10^{-14}, \alpha(4075)=0.640 \times$ $10^{-13}$. Accordingly we found 
Table 4. Abundance based on recombination lines.

\begin{tabular}{|c|c|c|c|c|c|c|c|c|c|c|}
\hline Ion & $\lambda_{\text {lab }}(\AA)$ & $\begin{array}{l}\text { Ref. } \\
\text { (Case) }\end{array}$ & a & $\mathrm{b}$ & c & d & $\mathrm{Br}$ & $\begin{array}{l}\alpha\left(\mathrm{J}, \mathrm{J}^{\prime}\right) \\
\left(\times 10^{-13}\right)\end{array}$ & I & $\mathrm{X}^{+} / \mathrm{H}^{+}$ \\
\hline $\mathrm{H} \beta$ & 4861 & $\mathrm{P}(\mathrm{B})$ & 0.668 & -0.507 & 1.221 & 0.653 & 1.000 & 0.317 & 100.000 & \\
\hline $\mathrm{H} \alpha$ & 6563 & $\mathrm{P}(\mathrm{B})$ & 2.708 & -0.648 & 1.315 & 0.523 & 1.000 & 1.240 & 234.154 & \\
\hline \multirow[t]{6}{*}{ HeI } & 4471 & $\mathrm{P}(\mathrm{A})$ & 0.429 & -0.530 & 1.505 & 0.779 & 0.790 & 0.144 & 4.769 & 0.0967 \\
\hline & 4922 & $\mathrm{P}(\mathrm{A})$ & 0.135 & -0.540 & 1.505 & 0.779 & 0.740 & 0.042 & 0.880 & 0.0666 \\
\hline & 4713 & $\mathrm{P}(\mathrm{A})$ & 0.019 & -0.518 & -0.045 & 0.951 & 0.626 & 0.013 & 0.631 & 0.1513 \\
\hline & 5876 & $\mathrm{P}(\mathrm{A})$ & 1.323 & -0.696 & 1.683 & 0.667 & 1.000 & 0.528 & 15.005 & 0.1089 \\
\hline & 7281 & $\mathrm{P}(\mathrm{A})$ & 0.036 & -0.503 & 0.165 & 0.614 & 1.000 & 0.032 & 0.854 & 0.1266 \\
\hline & Adopted & & & & & & & & & 0.1100 \\
\hline HeII & 4686 & $\mathrm{P}(\mathrm{B})$ & 1.549 & -0.693 & 2.884 & 0.609 & 1.000 & 0.428 & 2.533 & 0.0181 \\
\hline $\mathrm{CII}$ & 4267 & $\mathrm{P}(\mathrm{A})$ & 1.011 & -0.754 & 2.587 & 0.719 & 0.950 & 1.738 & 0.335 & $5.368 \times 10^{-4}$ \\
\hline \multirow[t]{3}{*}{ CIII } & 4649 & $\mathrm{P}(\mathrm{A})$ & 0.429 & -0.501 & 0.624 & 0.681 & 0.940 & 1.562 & 0.102 & $1.982 \times 10^{-4}$ \\
\hline & 8197 & $\mathrm{P}(\mathrm{A})$ & 0.587 & -0.780 & 7.448 & 0.720 & 1.000 & 0.455 & 0.151 & $1.775 \times 10^{-3}$ \\
\hline & Adopted & & & & & & & & & $1.982 \times 10^{-4}$ \\
\hline \multirow[t]{2}{*}{ NII } & 5679 & $\mathrm{P}(\mathrm{A})$ & 1.200 & -0.615 & 1.683 & 0.667 & 0.337 & 1.125 & 0.050 & $1.648 \times 10^{-4}$ \\
\hline & 5679 & $\mathrm{P}(\mathrm{B})$ & 1.462 & -0.628 & 1.683 & 0.667 & 0.337 & 1.371 & 0.050 & $1.351 \times 10^{-4}$ \\
\hline \multirow[t]{5}{*}{ NIII } & 4100 & $\mathrm{P}(\mathrm{A})$ & 1.350 & -0.734 & 0.624 & 0.681 & 0.058 & 0.359 & 0.462 & $3.445 \times 10^{-3}$ \\
\hline & 4100 & $\mathrm{P}(\mathrm{B})$ & 2.020 & -0.694 & 0.624 & 0.681 & 0.058 & 0.536 & 0.462 & $2.308 \times 10^{-3}$ \\
\hline & 4641 & $\mathrm{P}(\mathrm{A})$ & 1.434 & -0.707 & 1.683 & 0.667 & 0.005 & 0.020 & 0.863 & 0.1303 \\
\hline & 4641 & $\mathrm{P}(\mathrm{B})$ & 1.449 & -0.700 & 1.683 & 0.667 & 0.768 & 3.111 & 0.863 & $8.402 \times 10^{-4}$ \\
\hline & Adopted & & & & & & & & & $2.142 \times 10^{-3}$ \\
\hline \multirow[t]{5}{*}{ OII } & 4072 & $\mathrm{~L}(\mathrm{~A})$ & 0.876 & -0.735 & 0.137 & 0.062 & 0.865 & 0.640 & 0.300 & $1.246 \times 10^{-3}$ \\
\hline & 4075 & L(A) & 0.876 & -0.735 & 0.137 & 0.062 & 1.000 & 0.740 & 0.292 & $1.050 \times 10^{-3}$ \\
\hline & 4651 & $\mathrm{P}(\mathrm{A})$ & 1.257 & -0.624 & 1.683 & 0.667 & 1.000 & 3.997 & 0.173 & $1.314 \times 10^{-4}$ \\
\hline & 4651 & $\mathrm{P}(\mathrm{B})$ & 1.296 & -0.620 & 1.683 & 0.667 & 1.000 & 4.120 & 0.173 & $1.275 \times 10^{-4}$ \\
\hline & Adopted & & & & & & & & & $1.148 \times 10^{-3}$ \\
\hline OIII & 3762 & $\mathrm{P}(\mathrm{A})$ & 1.200 & -0.615 & 1.683 & 0.667 & 0.383 & 1.461 & 0.415 & $2.137 \times 10^{-3}$ \\
\hline
\end{tabular}

P: Péquignot et al (1991); L: Liu et al. (1995); (A): case A; (B): case B.

$$
\frac{\mathrm{O}^{2+}}{\mathrm{H}^{2+}}=\frac{4072}{4861} \times \frac{0.317 \times 10^{-13}}{0.640 \times 10^{-13}} \times \frac{0.300}{100}=1.246 \times 10^{-13}
$$

Table 4 lists abundances derived in a similar way, assuming that the permitted lines are emitted through the recombination mechanism. We calculated the abundances assuming the emissions are either Case A or Case B, depending on the availability of their

Table 5. NGC 6210 abundance comparison

\begin{tabular}{ccccc}
\hline \hline \multirow{2}{*}{ Element } & \multicolumn{4}{c}{$\mathrm{N}(\mathrm{X})$} \\
\cline { 2 - 5 } & Ours & $\mathrm{K}$ and $\mathrm{H}^{(1)}$ & $\mathrm{Mean}^{(2)}$ & $\mathrm{Sun}^{(3)}$ \\
\hline $\mathrm{He} / \mathrm{H}$ & 0.13 & 0.12 & 0.11 & 0.086 \\
$\mathrm{C} / \mathrm{H}$ & $8.15 \mathrm{e}-4$ & $9.24 \mathrm{e}-5$ & $6.48 \mathrm{e}-4$ & $2.57 \mathrm{e}-4$ \\
$\mathrm{~N} / \mathrm{H}$ & $2.57 \mathrm{e}-3$ & $1.45 \mathrm{e}-4$ & $1.40 \mathrm{e}-4$ & $6.31 \mathrm{e}-5$ \\
$\mathrm{O} / \mathrm{H}$ & $3.76 \mathrm{e}-3$ & $4.40 \mathrm{e}-4$ & $4.93 \mathrm{e}-4$ & $4.57 \mathrm{e}-4$ \\
\hline
\end{tabular}

(1) Kwitter and Henry (1998); (2) PN average by Kingsburgh and Barlow (1994); (3) Hyung and Feibelman (2004, reference therein) recombination coefficients. The former is for the optically thin, while the latter is optically thick. As mentioned earlier, the above derivation was carried out assuming that these lines are emitted through the recombination process.

Unfortunately, there is no systematic way other than the recombination mechanism to derive the chemical abundances from permitted emission lines. Thus, we will quote the result in Table 4 for the abundance anomaly problem in NGC 6210. The abundances given in Table 4 are, however, partial only for the observed ions. To correct for the unobserved ionic stages, we obtained the ionization correction factor with the photoionization model (using CLOUDY, Lee and Hyung, 2008). The elemental abundances for 3 elements are given in Table 5. In Table 5, we also presents the abundances derived from the forbidden lines by Kwitter and Henry (1998). com abundances 
derived from the permitted lines are much larger than those by Kwitter and Henry (1998), i.e., a factor of 10 for $\mathrm{C}, \mathrm{N}$, and $\mathrm{O}$. They are even larger than the average PN (Kingsburgh and Barlow, 1994) or the Solar values (see Table 5). Low to intermediate mass stars of $0.8-8 \mathrm{Mo}$ are known to end their lives without processing carbon and hy vier element burning. These stars experience both hydrogen and hylium burning during main-sequence, Giant Branch (GB), and Asymptotic Giant Branch (AGB), and they become PNs. The chemistry around AGB stars is controlled by the $\mathrm{C} / \mathrm{O}$ ratio. At the $\mathrm{AGB}$ phase, strong convection flows dredge up synthesized elements to the surface. Thus, an AGB star has an oxygen rich atmosphere. The triple-alpha process inside the star is producing ${ }^{12} \mathrm{C}$. This carbon is dredged up to the surface and thus changes the chemical composition of the atmosphere. If the carbon-to-oxygen ratio $(\mathrm{C} / \mathrm{O})$ is less than unity, carbon atoms will be locked into $\mathrm{CO}$ around this star. Hence the later stage PN will be oxygen-rich. cn the other hand, If the carbon processed in the hyliumburning regions of $\mathrm{AGB}$ is continuously dredged up, the surface of the star changes from an oxygen-rich $(\mathrm{C} / \mathrm{O}<1)$ environment to carbon-rich $(\mathrm{C} / \mathrm{O}>1)$.

Stars with masses $\mathrm{M}_{\text {star }}>4 \mathrm{M}_{\circ}$, in which the bottom of the convective envelope can reach temperatures $\mathrm{T}$ $>5 \times 10^{7} \mathrm{~K}$, can have hot envelope-burning. This burning, known as hot bottom burning, destroys ${ }^{12} \mathrm{C}$ and ${ }^{18} \mathrm{O}$ through the $\mathrm{CNO}$ cycle, which eventually yields nitrogen-rich Type I planetary nebulae (Peimbert, 1979; Kingsburgh and Barlow, 1994). The nitrogen abundance derived by Kwitter and Henry (1998) is comparable to the average $\mathrm{PN}$ value. Considering the nitrogen abundance. NGC 6210 is not perhaps evolved from that of Type I's high massive progenitor. Based on the helium and nitrogen abundances indicated by our HES and Kwitter and Henry (1998), we conclude that NGC 6210 might have been evolved from a relatively high mass progenitor, $3 \mathrm{Mo}$, or slightly higher. The line profile in Sec. 3 and recombination process studied here imply that the permitted lines are originated from other light sources, e.g., HeII or stellar UV fluorescence photon.

\section{Discussion}

We investigated the high dispersion HES optical data obtained with the $3 \mathrm{~m}$ telescope at Lick observatory. The high excitation HeII lines were observed in our observation, while Kwitter and Henry (1998) did not. Our high dispersion data allowed us to separate many diagnostically important lines, so we were able to derive the physical conditions and radial velocities, which were not possible in previous studies. The variation of expansion velocities indicates that the outflow gas got accelerated. The slope of the radial variation of velocities is not completely linear, which might be due to the precession of the nebular axis. Our HES data show many permitted lines. We also derived the chemical abundances of NGC 6210 based on the permitted lines, which are larger than the average PN or the Sun.

Because of a long exposure in the 1995 data, we were able to analyze the line profile and derived radial velocities from OII lines, which indicate a different origin from [OIII] lines. The OII and other recombination lines are likely to form through the fluorescence mechanism. Hence, the abundances found from these permitted lines do not represent the elemental abundance. The emission from permitted lines is merely the fluorescent light from the CSPN or other abundant elements, i.e., $\mathrm{H}$ or He. The enhancement of $\mathrm{He}$ is conspicuous. If some fraction of $\mathrm{C}, \mathrm{N}$, and $\mathrm{O}$ abundances are due to elements themselves, the heavy elements of NGC 6210 are enhanced comparing with the average PNs. AGB stars have circumstellar shells which are either C-rich or O-rich, and NGC 6210 is a remnant of the O-rich AGB outflow material. Although the Galactic latitude is relatively high, i.e., $b=37^{\circ}$, the enhanced elemental abundances indicate that the progenitor was born in the Galactic plane. The enhanced He abundances or $\mathrm{N}$ abundances imply that NGC 6210 is a Peimbert type II PN, perhaps evolved from a star $>3 \mathrm{Mo}$.

Our HES data secured over two bright rims (NE and $\mathrm{N}$ ) seem to represent relatively high temperature zone close to the central region, while Kwitter and 
Henry (1998) and IUE data were for the whole object. As mentioned earlier, the elements around AGB stars are controlled by the $\mathrm{C} / \mathrm{O}$ ratio. NGC 6210 is evolved from an O-rich AGB or M-type AGB star. Our discussion on chemistry and elemental abundance will be speculative till the further IR and UV data are supplemented, though. Other elemental abundances must be carefully investigated using the weakly registered forbidden lines in the future study.

\section{Acknowledgment}

This work was supported by the research grant of Chungbuk National University in 2009. S.H. is grateful to the late Prof. Lawrence H. Aller of UCLA, who carried out the Hamilton Echelle observation program together with him.

\section{References}

Acker, A., Marcout, J., Ochsenbein, F., Stenholm, B., and Tylenda, R., 1992, Strasbourg-ESO catalogue. 196-200.

Aller, L.H., 1984, Physics of thermal gaseous nebulae. Reidel Publisher Company, Dordrecht, Holland, 350 p.

Choi, Y., Lee, S.-J., and Hyung, S., 2008, Expansion velocity investigation of the elliptical planetary nebula NGC 6803. Journal of the Korean Astronomical Society, 41, 163-172.

Hyung, S., 1994, Density contrast shell models for the planetary nebula IC 2165, The Astrophysical Journal Supplement Series, 90, 119-148.

Hyung, S. and Feibelman, W.A., 2004, Optical and IUE spectra of the planetary nebula NGC 7026. The Astrophysical Journal, 614, 745-756.

Kingsburgh, R.L. and Barlow, M.J., 1994, Elemental abundances for a sample of southern galactic planetary nebulae. Monthly Notices of Royal Astronomical Society, 271, 257-299.

Kwitter, K.B. and Henry, R.B.C., 1998, A new look at carbon abundances in planetary nebulae III. DdDm 1, IC 3568, IC 4593, NGC 6210, NGC 6720, NGC 6826, and NGC 7009. The Astrophysical Journal, 493, 247259 .
Lee, S.-J. and Hyung, S., 2008, Photoionization models for planetary nebulae: Comparison of predictions by NEBULA and CLOUDY. Journal of Korean Earth Science Society, 29, 5, 419-427.

Lee, S.-M. and Hyung, S., 2007, Kinematics and geometrical structure of the planetary nebula NGC 6881. Journal of Korean Earth Science Society, 28, 847-856.

Liu, Y., Liu, X.-W., Barlow, M.J., and Luo, S.-G., 2004, Chemical abundances of planetary nebulae from optical recombination lines-II. Abundances derived from collisionally excited lines and optical recombination lines. Monthly Notices of the Royal Astronomical Society, 353, 1251-1285.

Liu, X.-W., Storey, P.J., Barlow, M.J., and Clegg, R.E.S., 1995, The rich OII recombination spectrum of the planetary nebula NGC 7009: New observations and atomic data. Monthly Notices of the Royal Astronomical Society, 1995, 272, 369-388.

Mendez, R.H., 1991, Photospheric abundances in central stars of planetary nebulae, and evolutionary implications. International Astronomical Union, 145, 375-386.

Osterbrock, D.E., 1989, Astrophysics of gaseous nebulae and active galactic nuclei. University Science Books, Sausalito, California, USA, 408 p.

Peimbert, M., 1979, Chemical evolution of the galactic interstellar medium-Abundance gradients. International Astronomical Union, 84, 307-315.

Péquignot, D., Petitjean, P., and Boisson, D., 1991, Total and effective radiative recombination coefficients. Astronomy and Astrophysics, 251, 680-688.

Phillips, J.P. and Cuesta, L., 1996, NGC 6210: An observational case study of a jet emitting source. The Astronomical Journal, 111, 1227-1240.

Pottasch, S.R., Bernard-Salas, J., and Roelling, T.L., 2009, Abundances in the planetary nebula NGC 6210. Astronomy and Astrophysics, 499, 249-256.

Pottasch, S.R., 1984, Planetary nebulae (Dordrecht: Reidel). 44-77.

Sabbadin, F., Turatto, M., Ragazzoni, R., and Benetti, S., 2006, The structure of planetary nebulae: Theory vs. practice. Astronomy and Astrophysics, 451, 937-949.

Sabbadin, F., Turatti, M., Cappellaro, E., Benetti, S., and Ragazzoni, R., 2004, The 3-D ionization structure and evolution of NGC 7009 (Saturn Nebula). Astronomy and Astrophysics, 416, 955-981.

Seaton, M.J., 1979, Instellar extinction in the UV. Monthly Notices of Royal Astronomical Society, 187, 73-76.

Manuscript received: July 15, 2009

Revised manuscript received: August 29, 2009

Manuscript accepted: September 8, 2009 